\title{
Gastric cancer: immunohistochemical classification of molecular subtypes and their association with clinicopathological characteristics
}

\author{
Eva-Maria Birkman $^{1,2}$ (D) - Naziha Mansuri ${ }^{1} \cdot$ Samu Kurki $^{3}$ • Annika Ålgars ${ }^{4,5}$. \\ Minnamaija Lintunen $^{2} \cdot$ Raija Ristamäki $^{4}$. Jari Sundström ${ }^{1,2}$ • Olli Carpén ${ }^{1,3,6}$
}

Received: 25 May 2017 / Revised: 5 September 2017 / Accepted: 25 September 2017 /Published online: 19 October 2017

(C) The Author(s) 2017. This article is an open access publication

\begin{abstract}
Gastric cancer is traditionally divided into intestinal and diffuse histological subtypes, but recent molecular analyses have led to novel classification proposals based on genomic alterations. While the intestinal- and diffuse-type tumours are distinguishable from each other at the molecular level, intestinal-type tumours have more diverse molecular profile. The technology required for comprehensive molecular analysis is expensive and not applicable for routine clinical diagnostics. In this study, we have used immunohistochemistry and in situ hybridisation in molecular classification of gastric adenocarcinomas with an emphasis on the intestinal subtype. A tissue microarray consisting of 244 gastric adenocarcinomas was constructed, and the tumours were divided into
\end{abstract}

Virchows Archiv conforms to the ICMJE recommendation for qualification of authorship. The ICMJE recommends that authorship be based on the following four criteria:

- Substantial contributions to the conception or design of the work; or the acquisition, analysis, or interpretation of data for the work; AND

- Drafting the work or revising it critically for important intellectual content; AND

- Final approval of the version to be published; AND

- Agreement to be accountable for all aspects of the work in ensuring that questions related to the accuracy or integrity of any part of the work are appropriately investigated and resolved.

Eva-Maria Birkman and Naziha Mansuri have equal contribution.

Eva-Maria Birkman

emabir@utu.fi

1 Department of Pathology, University of Turku, Kiinamyllynkatu 10, 20520 Turku, Finland

2 Department of Pathology, Turku University Hospital, Kiinamyllynkatu 10, 20520 Turku, Finland

3 Auria Biobank, University of Turku and Turku University Hospital, Kiinamyllynkatu 8, 20520 Turku, Finland four subgroups based on the presence of Epstein-Barr virus, TP53 aberrations and microsatellite instability. The intestinaland diffuse-type tumours were separately examined. The distribution of EGFR and HER2 gene amplifications was studied in the intestinal-type tumours. Epstein-Barr virus positive intestinal-type tumours were more common in male patients ( $p=0.035$ ) and most often found in the gastric corpus $(p=0.011)$. The majority of the intestinal-type tumours with TP53 aberrations were proximally located $(p=0.010)$. All tumours with microsatellite instability showed intestinal-type histology $(p=0.017)$ and were associated with increased overall survival both in the univariate $(p=0.040)$ and multivariate analysis $(p=0.015)$. In conclusion, this study shows that
4 Department of Oncology, Turku University Hospital, Hämeentie 11, 20520 Turku, Finland

5 MediCity Research Laboratory, University of Turku, Tykistökatu 6, 20520 Turku, Finland

6 Pathology, Research Programs Unit and HUSLAB, University of Helsinki and Helsinki University Hospital, Haartmaninkatu 3, 00014 Helsinki, Finland 
gastric adenocarcinomas can be classified into biologically and clinically different subgroups by using a simple method also applicable for clinical diagnostics.

Keywords Gastric cancer · Immunohistochemistry · In situ hybridisation $\cdot$ Molecular classification

\section{Introduction}

Gastric cancer is one of the major causes of cancer-related death worldwide [1]. Gastric adenocarcinomas have traditionally been divided into intestinal and diffuse subtypes according to the Laurén classification based on the histological characteristics of the tumours [2]. However, The Cancer Genome Atlas Consortium (TCGA) has recently proposed a molecular subtyping of gastric adenocarcinomas based on the presence of Epstein-Barr virus (EBV), microsatellite instability (MSI), genomic stability (GS) and chromosomal instability (CIN) [3]. The majority of the GS tumours have diffuse histology, while the other subgroups contain predominantly intestinal-type tumours. An alternative classification has been proposed by the Asian Cancer Research Group (ACRG). This proposal stratifies gastric adenocarcinomas into tumours with MSI, microsatellite-stable tumours showing epithelial to mesenchymal transition (MSS/EMT), MSS tumours with intact TP53 activity (MSS/TP53+) and MSS tumours with functional loss of TP53 (MSS/TP53-) [4]. These classification systems have provided valuable information about the variability in biological characteristics among gastric adenocarcinomas. Instead of considering gastric cancer as a single disease, it has also become clear that when exploring new cancer therapies, future studies need to be conducted among defined sets of patients whose tumours have specific genomic abnormalities. In clinical practice, HER2 is the only predictive biomarker for targeted therapy currently used for patient selection in gastric cancer.

The complex methodologies used in these abovementioned studies are not applicable for routine clinical diagnostics, and some more straightforward methods have already been proposed [5-8]. In this study, we have constructed a nextgeneration tissue microarray (ngTMA) from 244 intestinaland diffuse-type adenocarcinomas of the stomach, gastrooesophageal junction (GOJ) and distal oesophagus [9]. Using this cohort, we have been able to identify four subgroups of tumours with distinct molecular and clinicopathological characteristics by combining the Laurén classification, MSI and TP53 immunohistochemistry (IHC) and EBER in situ hybridisation (ISH). Additionally, the analysis of Ecadherin expression was performed in order to compare our method with the previously published studies, and the distribution of EGFR and HER2 gene amplifications were examined among these subgroups in the intestinal-type tumours.

\section{Materials and methods}

\section{Patients and tumour specimens}

The study population consists of 244 patients diagnosed with adenocarcinoma of the stomach, GOJ or distal oesophagus at the Turku University Hospital between years 1993 and 2012. The initial search in the clinical database of Auria Biobank retrieved tumour specimens from 437 patients. The exclusion criteria were carcinoma in situ (Tis, $n=23$ ), insufficient sample material or indeterminate histology $(n=63)$, neuroendocrine histology $(n=3)$ and metastatic adenocarcinoma from a different organ $(n=6)$. This resulted in 190 patients with intestinal-type tumours and 152 patients with diffuse-type tumours. From these, all intestinal-type tumours and 54 representative diffuse-type tumours were included in the ngTMA. Among all of the patients, 11.9\% (29/244) did receive preoperative chemotherapy (13 patients with intestinal-type and 16 with diffuse-type tumours). The type of surgery was total gastrectomy for $154(63.1 \%)$ patients, subtotal gastrectomy or tumour resection for 72 (29.5\%) patients and palliative surgery for $18(7.4 \%)$ patients. The extent of surgery was determined as R0 (no residual tumour) for 180 (73.8\%) patients, R1 (microscopic residual tumour) for 34 (13.9\%) patients and R2 (macroscopic residual tumour) for $20(8.2 \%)$ patients. The extent of surgery could not be determined for $10(4.1 \%)$ patients. Tumour stage was assessed according to the current WHO Classification manual [10]. The median follow-up time of the patients was 125 months. All corresponding H\&E slides have been reviewed for confirmation of diagnosis and adequacy of material. Relevant clinical information has been gathered from each case. The reporting of the study has been performed in compliance with the current recommendations [11]. The patient characteristics are presented in Table 1.

\section{Tissue microarray construction}

The ngTMA was created as follows [9]. The appropriate formalin-fixed paraffin-embedded (FFPE) tissue specimens were chosen based on clinical data and retrieved from the pathology archives. A representative haematoxylin-eosin (H\&E) section containing areas of invasive carcinoma was selected from each tumour. New H\&E slides were produced, scanned (Pannoramic P250, 3DHistech) and uploaded into the university digital microscopy web portal (casecenter.utu.fi). Each slide was viewed using Pannoramic Viewer software (3DHistech). Using the 1.0-mm annotation tool, annotations of different colours corresponding to various histological areas were placed onto each digital slide. Two annotations were placed in the centre of the tumour and two annotations in the periphery or invasive front of the tumour. The corresponding tissue cores were then transferred into the TMA blocks using an automated TMA instrument (TMA 
Table 1 Patient characteristics

\begin{tabular}{|c|c|c|c|}
\hline Number of patients & All, $n(\%)$ & Intestinal, $n(\%)$ & Diffuse, $n(\%)$ \\
\hline All & 244 & $190(77.9)$ & $54(22.1)$ \\
\hline \multicolumn{4}{|c|}{ Median age at diagnosis (range) } \\
\hline & $72.3(32.9-90.9)$ & $74.4(32.9-90.9)$ & $66.8(36.9-85.1)$ \\
\hline \multicolumn{4}{|l|}{ Patient sex } \\
\hline Female & $101(41.4)$ & $68(35.8)$ & $33(61.1)$ \\
\hline Male & $143(58.6)$ & $122(64.2)$ & $21(38.9)$ \\
\hline \multicolumn{4}{|l|}{ Site of primary tumour ${ }^{\mathrm{a}}$} \\
\hline Distal oesophagus & $19(7.8)$ & $19(10.0)$ & \\
\hline GOJ/cardia & $60(24.6)$ & $60(31.6)$ & \\
\hline Corpus & $106(43.4)$ & $52(27.4)$ & \\
\hline Antrum/pylorus & $59(24.2)$ & $59(31.1)$ & \\
\hline \multicolumn{4}{|l|}{ Tumour differentiation grade } \\
\hline Grade 1 & $17(7.0)$ & $17(8.9)$ & $0(0)$ \\
\hline Grade 2 & $93(38.1)$ & $93(48.9)$ & $0(0)$ \\
\hline Grade 3 & $134(54.9)$ & $80(42.1)$ & $54(100.0)$ \\
\hline \multicolumn{4}{|l|}{ Stage } \\
\hline I & $46(18.9)$ & $40(21.1)$ & $6(11.1)$ \\
\hline II & $102(41.8)$ & 79 (41.6) & $23(42.6)$ \\
\hline III & $83(34.0)$ & $61(32.1)$ & $22(40.7)$ \\
\hline IV & $13(5.3)$ & $10(5.3)$ & $3(5.6)$ \\
\hline \multicolumn{4}{|l|}{ Follow-up status } \\
\hline Alive and free of disease & $48(19.7)$ & $34(17.9)$ & $14(25.9)$ \\
\hline Alive with disease & $1(0.4)$ & $1(0.5)$ & $0(0)$ \\
\hline Deceased & 195 (79.9) & 155 (81.6) & $40(74.1)$ \\
\hline
\end{tabular}

GOJ gastro-oesophageal junction

${ }^{\text {a }}$ Diffuse-type tumours are included in the tumours of the corpus

Grandmaster, 3DHistech) by overlaying each annotated digital slide with the corresponding tissue specimen. One tissue core containing benign tissue was selected from each tumour to act as a control. The constructed TMA blocks were sectioned, stained, scanned and uploaded into the web portal (casecenter.utu.fi), and each individual spot was scored by two pathologists (EB and NM). The resulting scores were combined with the clinical data for statistical analysis.

The link https://seafile.utu.fi/d/7c4aa1964b/ contains examples of our TMA results. The directory "TMA staining" contains low resolution images of all stainings performed on TMA block number 7, and "TMA map and score" contains the map and scores of the same TMA block. The directory "Example scoring" contains selected high resolution images of positive/negative and aberrant/wildtype stained tissue cores.

\section{Immunohistochemistry and in situ hybridisation}

IHC reactions were performed on 4- $\mu$ m paraffin sections of each tumour on the TMA slides with BenchMark XT (Ventana/Roche). For TP53, a ready-to-use antibody clone
Bp53-11 (Ventana/Roche) was used, and the protocol included mild ( $30 \mathrm{~min}$ ) $\mathrm{CC} 1$ pretreatment together with 28 -min antibody incubation. Signal detection was performed with ultraView universal DAB Detection Kit (Ventana/Roche). For MLH1, an antibody clone G168-15 (BD Pharmingen) was used at 1:5 dilution together with standard $(60 \mathrm{~min})$ $\mathrm{CC} 1$ pretreatment and 36-min antibody incubation. The signal was detected with ultraView Universal DAB Detection Kit and amplification kit. For MSH2, an antibody clone G2191129 (BD Pharmingen) was used at 1:200 dilution together with standard $\mathrm{CC} 1$ pretreatment and 28-min antibody incubation. The signal was detected with ultraView Universal DAB Detection Kit. For MSH6, an antibody clone EP49 (Epitomoc) was used at 1:200 dilution together with standard $\mathrm{CC} 1$ pretreatment and 32-min antibody incubation. The signal was detected with ultraView Universal DAB Detection Kit. For PMS2, a ready-to-use antibody clone EPR3947 (Ventana/ Roche) was used together with extended (90 min) CC1 pretreatment, 44-min antibody incubation and 12-min HQ LINKER and HRP MULTIMER enhancements. The signal was detected with OptiView Universal DAB Detection Kit and amplification kit. For EBV, a ready-to-use EBER (EBV- 
encoded small RNA) probe (Ventana/Roche) was used together with ISH-Protease 3 pretreatment for $28 \mathrm{~min}$ and 1-h probe incubation. The signal was detected with ISH iVIEW Blue Detection Kit. For E-cadherin, an antibody clone NCH-38 (Agilent Technologies) was used at 1:100 dilution together with standard CC1 pretreatment and 32-min antibody incubation. The signal was detected with ultraView Universal DAB Detection Kit and amplification kit. A complete loss of or strong diffuse TP53 nuclear positivity was classified as aberrant TP53 expression. EBER ISH was scored either positive or negative according to the nuclear reaction. A tumour was classified as MSI if at least one of the markers (MLH1, MSH2, MSH6 and PMS2) showed a complete loss of nuclear reactivity together with positive background reaction in benign epithelium, smooth muscle cells and lymphocytes. Negative nuclear reactivity with negative background was considered controversial and not used for classification Loss of membranous reactivity or only faint cytoplasmic reaction was classified as aberrant E-cadherin expression (Fig. 1a). The methods for EGFR and HER2 IHC and silver in situ hybridisation (SISH) have been described previously [12-15]. In short, with EGFR the scoring was based on the most intense membranous or membranous+cytoplasmic staining ( 0 , negative; $1+$, weak; $2+$, moderate; $3+$, strong). Specimens were classified as IHC high if showing $2+$ or $3+$ membranous or membranous+cytoplasmic staining intensity in $\geq 10 \%$ of tumour cells. With HER2 IHC, specimens showing $2+$ or $3+$ membranous staining in $\geq 10 \%$ of tumour cells were classified as IHC high. The IHC high samples were further analysed with SISH. The EGFR and HER2 IHC and SISH were performed on whole tissue sections.

\section{Statistical analysis}

Statistical analyses were performed with IBM SPSS Statistics for Windows, version 21.0 (IBM Corporation, Armonk, NY). Frequency table data were analysed using Pearson's $\chi^{2}$ test or Fisher's exact test for categorical variables and $2 \times 2$ tables were used to calculate odds ratios (OR). The Kaplan-Meier method and log-rank test as well as Cox's proportional hazards regression model were used for univariate survival analysis. Multivariate survival analysis was performed by Cox's proportional hazards regression model. Recurrence-free survival (RFS) was calculated from the time of diagnosis to the time of first recurrence, death of any cause or to the last follow-up date. Only recurrences $\geq 6$ months after the time of diagnosis were considered relevant. Detection of a local or distant recurrence $<6$ months from the diagnosis was considered likely to present an initially advanced disease. Patients without disease recurrence $\geq 6$ months after diagnosis were considered curatively treated. Overall survival (OS) was calculated from the time of diagnosis to the time of death of any cause or the last follow-up date. Five patients (2.0\%) who had received trastuzumab treatment for recurrent cancer were excluded from the OS analysis and additionally 13 patients with stage IV disease (5.3\%) from the RFS analysis. All statistical tests were two-sided, and $p$ values under 0.05 were considered statistically significant.

\section{Results}

\section{MSI, TP53 and E-cadherin immunohistochemistry and EBV in situ hybridisation}

EBV, MSI and TP53 were analysed in 238 tumours and Ecadherin in 232 tumours. In the other tumours, the markers could not be evaluated due to insufficient tissue material. EBV RNA was found to be present in 17/186 (9.1\%) of the intestinal tumours, while none of the diffuse tumours was EBV positive (Fisher's exact test, $p=0.028$; RR $0.91,95 \%$ CI $0.87-0.95)$. MSI was detected in 19/186 (10.2\%) of the intestinal tumours, while none of the diffuse tumours was found to have MSI phenotype (Fisher's exact test, $p=0.017$; RR 0.90, 95\% CI 0.86-0.94). Aberrant TP53 expression was observed to be significantly more common among intestinal-type (103/ $186,55.4 \%)$ than diffuse-type tumours $(10 / 52,19.2 \%)$ (Fisher's exact test, $p<0.0001$; OR $5.21,95 \%$ CI $2.47-$ 11.0). Ninety-four tumours (39.5\%) were found to be EBV negative, MSS and TP53 wild-type. Of these, 52/186 (28.0\%) tumours had intestinal-type and 42/52 (80.8\%) tumours had diffuse-type histology (Fisher's exact test, $p<0.0001$; OR $0.09,95 \%$ CI $0.04-0.20$ ). None of the MSI tumours had both EBV positivity and aberrant TP53 expression. Among the intestinal-type tumours, $3 / 183(1.6 \%)$ tumours had aberrant E-cadherin expression, whereas among the diffuse-type tumours, aberrant E-cadherin expression could be seen in 25/ 49 (51.0\%) tumours. Among the EBV negative, MSS and TP53 wild-type tumours, 21/39 (53.8\%) of the diffuse-type tumours but none of the intestinal-type tumours $(n=51)$ had aberrant E-cadherin expression.

Among the intestinal-type tumours, aberrant TP53 expression was more common in EBV negative than in EBV positive tumours (Fisher's exact test, $p<0.0001$; OR 0.041, 95\% CI 0.01-0.32). Tumours with aberrant TP53 expression were also more frequently MSS than MSI (Fisher's exact test, $p=0.003$; OR 5.46, 95\% CI 1.74-17.2). No association was found between aberrant E-cadherin expression and either EBV, TP53 or MSI status. Among the diffuse-type tumours, testing for statistical significance was not applicable for EBV or MSI (Table 2, Fig. 1b).

\section{EGFR and HER2 in the intestinal-type adenocarcinomas}

EGFR and HER2 protein expression levels were evaluated in 183 intestinal-type adenocarcinomas. Moderate/ 
Fig. 1 Classification of adenocarcinomas of the stomach, gastro-oesophageal junction and distal oesophagus based on immunohistochemistry and in situ hybridisation. a Examples of EBER in situ hybridisation and MLH1, TP53 and E-cadherin immunohistochemistry in eight oesophagogastric

adenocarcinomas. (a) EBV

positive, (b) TP53 aberration, (c) MLH1 mutated, (d) E-cadherin wild-type, (e) EBV negative, (f) TP53 wild-type, (g) MLH1 wildtype and (h) E-cadherin aberration. $\mathbf{b}$ The classification of the intestinal- and diffuse-type adenocarcinomas of the stomach, gastro-oesophageal junction and distal oesophagus according to the immunohistochemical data and in situ hybridisation. $\mathbf{c}$ Distribution of the four molecular subtypes of intestinal-type oesophagogastric adenocarcinomas in different anatomical locations.

${ }^{a}$ Immunohistochemical data available for 238 (EBV, MSI, TP53) or 232 tumours (Ecadherin). ${ }^{\mathrm{b}}$ Including one tumour (2\%) with co-amplification $E G F R$ and HER2. ${ }^{\circ}$ Including five tumours $(5 \%)$ with coamplification of $E G F R$ and HER2. EBV Epstein-Barr virus, GOJ gastro-oesophageal junction, MSS microsatellite-stable, MSI microsatellite-instable, wt wildtype, aberr aberration, amp amplification a

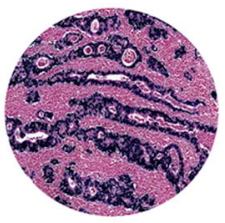

a

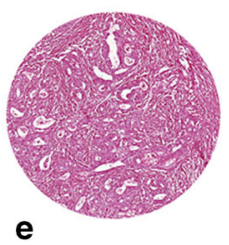

b
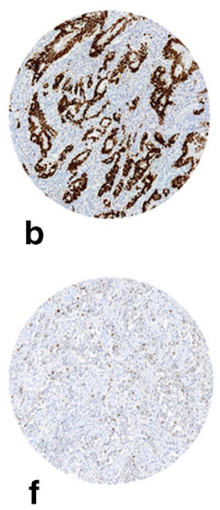

c

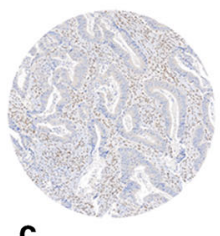

d
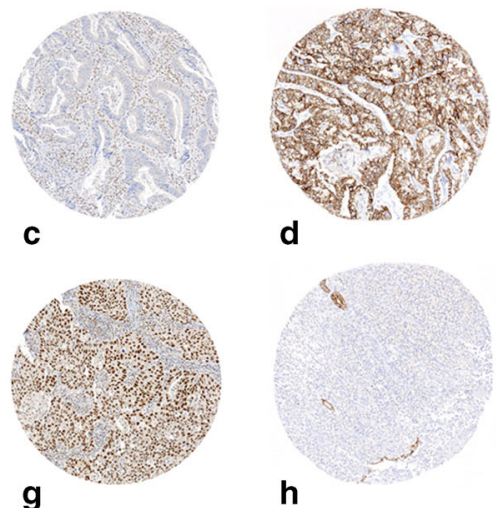

h

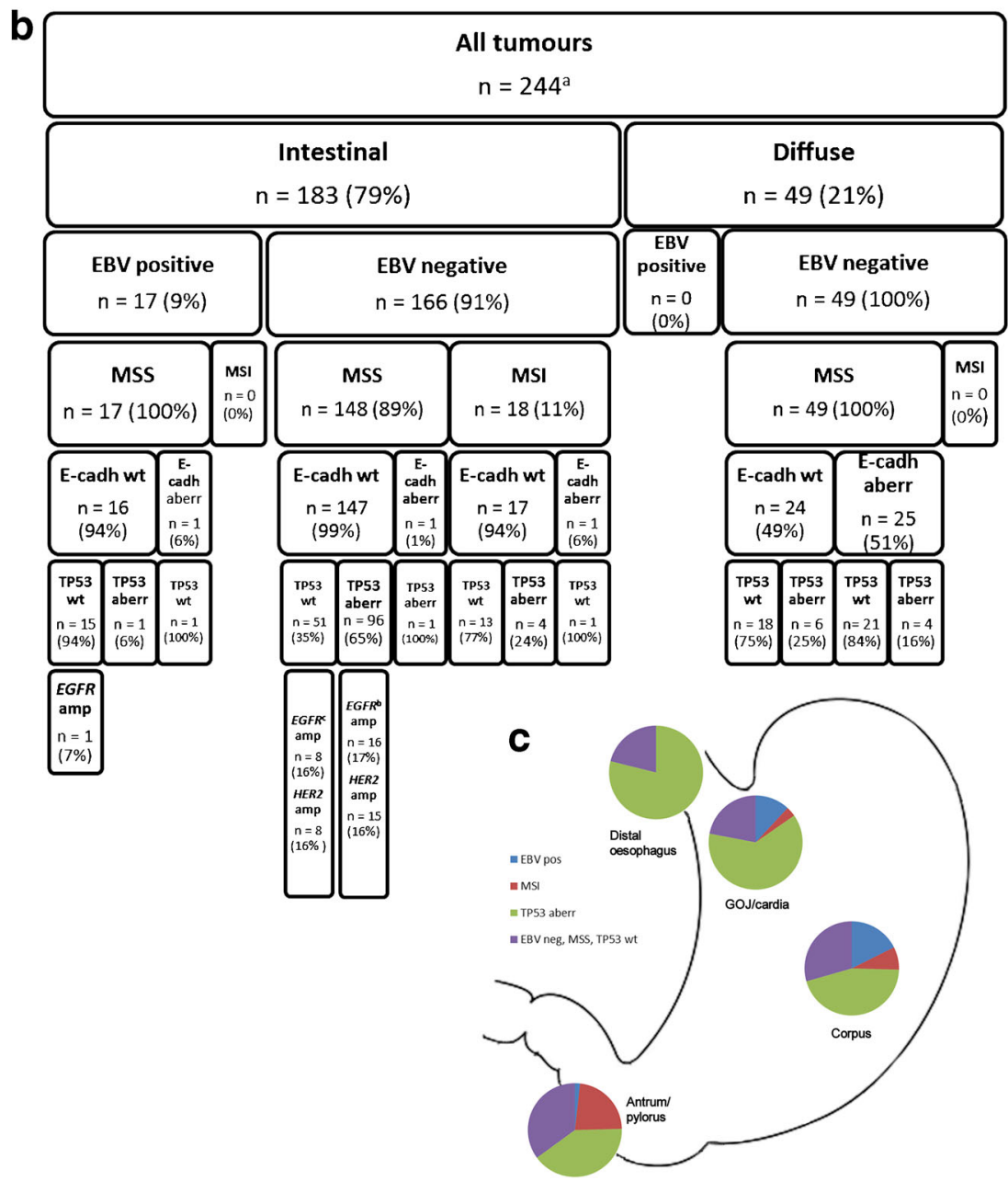

strong EGFR protein expression was found in 59 (32.2\%) of the tumours and $25(13.7 \%)$ of the tumours had moderate/strong HER2 protein expression. Among these, EGFR gene amplification was detected in 27/59 (14.8\% of the whole study material) tumours and HER2 gene amplification in 24/25 (13.1\% of the whole study material) tumours. No significant associations were observed between EGFR/HER2 protein expression level or gene amplification and TP53/EBV/MSI status. The majority of the EGFR $(n=17)$ or HER2 $(n=15)$ gene amplifications were found in the group of EBV negative and MSS tumours with aberrant TP53. Most of the co-amplifications $(n=5)$ were also found in this subgroup (Fig. 1b). The co-localisation of aberrant TP53 expression and either EGFR or HER2 gene amplification was detected more often in the proximal (distal oesophagus/GOJ/cardia) than distal (corpus/antrum/pylorus) intestinal-type tumours (Fisher's exact test, $p=0.019$; OR $2.83,95 \%$ CI 1.21-6.61). 


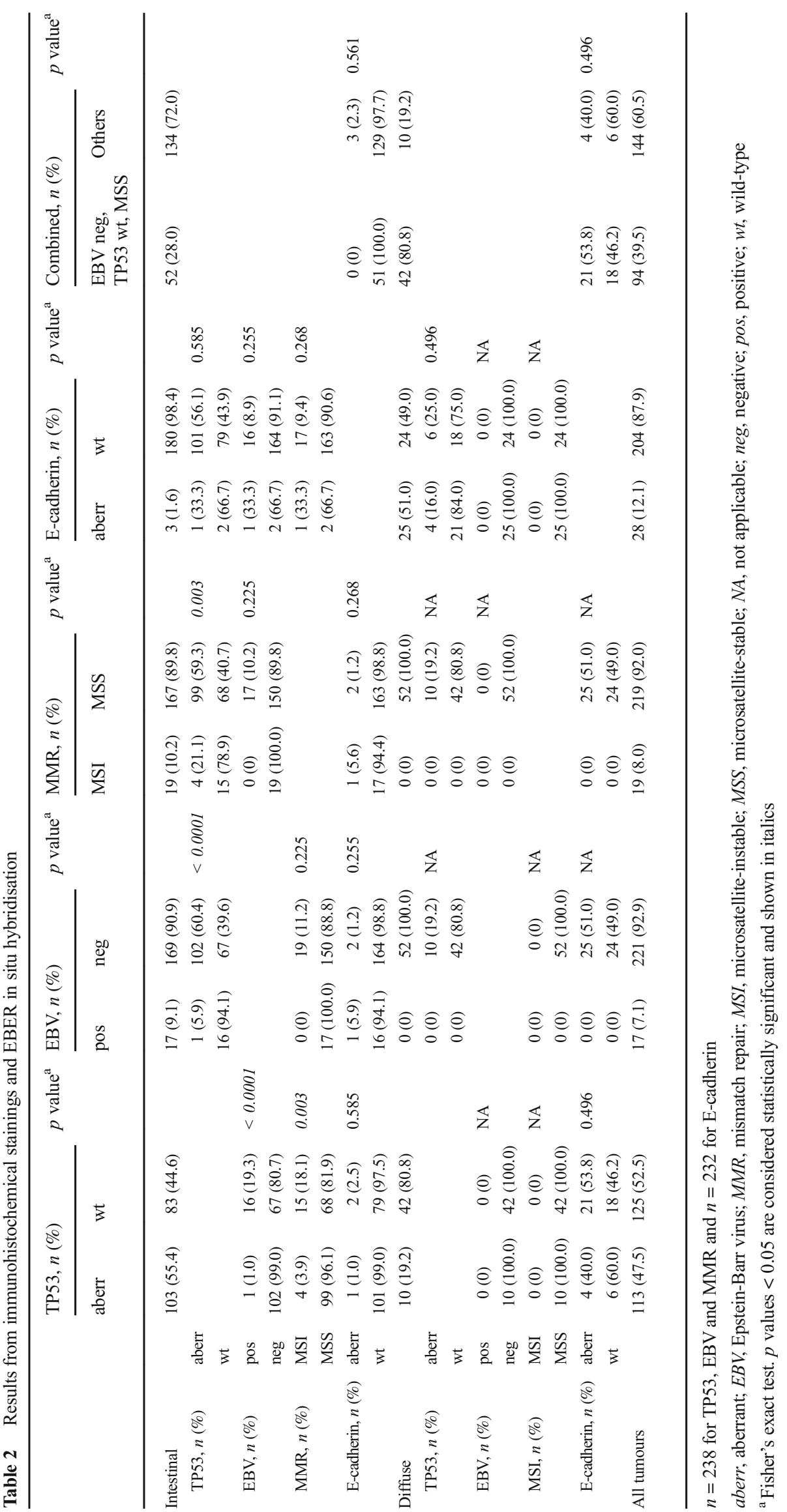




\section{TP53, EBV and MSI status in relation to clinicopathological variables}

Among the intestinal-type tumours, aberrant TP53 expression was more frequent in proximal than distal tumours (Fisher's exact test, $p=0.002$; OR 2.71, 95\% CI 1.47-5.00). In contrast, MSI phenotype was more frequent in distally located tumours (Fisher's exact test, $p=0.003$; OR 7.10, 95\% CI 1.59-31.7). EBV positive tumours were more common among male than female patients (Fisher's exact test, $p=0.035$; OR 4.57, 95\% CI 1.01-20.6), whereas MSI tumours were more common among female than male patients (Fisher's exact test, $p=0.042$; OR 2.89 , 95\% CI 1.07-7.36). EBV positive tumours were also more often poorly differentiated than well or moderately differentiated (Fisher's exact test, $p<0.0001$; OR $0.08,95 \%$ CI $0.02-0.37$ ) and most often located in the gastric corpus (Fisher's exact test, $p=0.011$ ). No significant associations were observed between the EBV negative/MSS/ TP53 wild-type tumours and the examined clinicopathological variables. Among diffuse-type tumours, no significant associations were found with the examined variables (Table 3).

\section{TP53, EBV and MSI status in relation to survival}

In univariate survival analysis for intestinal tumours, the presence of MSI was associated with longer overall survival (OS, median) (124.6 versus 28.7 months, log-rank test, $p=0.040$; Cox test, $p=0.043$, HR 0.54, 95\% CI 0.30-0.98) but not with recurrence-free survival (RFS). In intestinal tumours, increasing depth of tumour invasion was associated with shorter RFS and OS (RFS log-rank test, $p=0.045$; Cox test, $p=0.046$, HR 1.53, 95\% CI 1.01-2.32; OS log-rank test, $p=0.030$; Cox test, $p=0.031$, HR 1.54, 95\% CI 1.04-2.28). Similarly, increasing tumour stage was associated with shorter RFS and OS (RFS log-rank test, $p=0.019$; Cox test, $p=0.020$, HR 1.56, 95\% CI 1.07-2.26; OS log-rank test, $p<0.0001$; Cox test $p<0.0001$, HR 1.84, 95\% CI 1.32-2.57). In addition, patient age above median at the time of diagnosis was associated with shorter RFS and OS (RFS log-rank test, $p=0.006$; Cox test, $p=0.006$, HR 1.67, 95\% CI 1.16-2.42; OS log-rank test, $p=0.026$; Cox test $p=0.027$, HR 1.46, 95\% CI 1.04-2.03). No significant associations were observed between TP53 or EBV status and survival.

The multivariate model for OS included the following variables: patient age at diagnosis (below versus above median, median 72.3 years), postoperative T (T1-T2 versus T3-T4), tumour stage (I-II versus III-IV) and MMR status (MSS versus MSI, for intestinal tumours only). Among intestinal tumours, MSI status was found to be predictive for longer OS (Cox test, $p=0.015$, HR 0.46, 95\% CI 0.25-0.86) while patient age above median (Cox test, $p=0.009$, HR 1.57, 95\% CI 1.12-2.21) and increasing tumour stage (Cox test, $p=0.036$, HR $1.50,95 \%$ CI 1.03-2.18) were predictive for shorter OS. In diffuse-type tumours, patient age above median remained as a single predictive factor for shorter OS (Cox test, $p=0.030$, HR 2.29, 95\% CI 1.08-4.83) (Table 4).

\section{Discussion}

In this study, we describe a straightforward method for molecular classification of gastric cancer applicable for both clinical diagnostics and research purposes. With an emphasis on the intestinal-type adenocarcinomas, we have used IHC and ISH to define four subgroups of gastric adenocarcinomas with distinct molecular and clinical characteristics.

The recent molecular profiling studies have mainly been conducted in either Western [5] or Asian populations [4, 6-8]. The TCGA study contains patients from various geographical regions and among them they did not find any significant difference in the prevalence of the different molecular subtypes between the East-Asian group (Vietnam and South Korea) and the overall group [3]. Among the intestinal-type tumours, the frequency of EBV positive adenocarcinomas in our Finnish study population that represents Caucasian genetic background was $9.1 \%$, which is in line with the results of $3.0-9.5 \%$ from other studies showing no clear difference between the geographical regions $[3,4,6,8]$. The frequency of intestinal-type MSI tumours was $10.2 \%$ in our cohort which is similar to $9.2 \%$ found by Kim et al. (2016) but somewhat less than 24.5-26.0\% found in other studies with mixed patient populations [3, 4]. The presence of aberrant TP53 expression was found in $55.4 \%$ of the intestinal-type tumours in our study. This is close to the $53.1 \%$ of the TCGA study but somewhat different from the $31.3 \%$ found by Cristescu et al. (2015) or $67.3 \%$ by Kim et al. (2016). Still, no clear difference can be seen between the different geographical regions.

The molecular analyses of gastric adenocarcinomas have shown that the intestinal- and diffuse-type tumours are distinguishable from each other also at the molecular level, and the intestinal-type tumours have more diverse molecular profiles than the diffuse-type tumours, which are the predominant subtype in the "genomically stable" category [3, 4]. Therefore, the intestinal-type adenocarcinomas have been the main focus of our analyses, and a subset of diffuse-type tumours has served as a reference group for other publications. We have shown that the intestinal-type tumours rarely contain aberrant E-cadherin expression; and that by beginning with the Laurén classification, we could concentrate $25 / 28$ (89.3\%) of the tumours with aberrant E-cadherin expression into the diffuse subgroup. Notably, none of the EBV negative, MSS and TP53 wt intestinal-type tumours showed aberrant E-cadherin expression. This suggests that some other biomarker could be more specific than E-cadherin in detecting the intestinal-type tumours not characterised by EBV positivity, MSI or TP53 aberrations. In all, these observations imply that the 


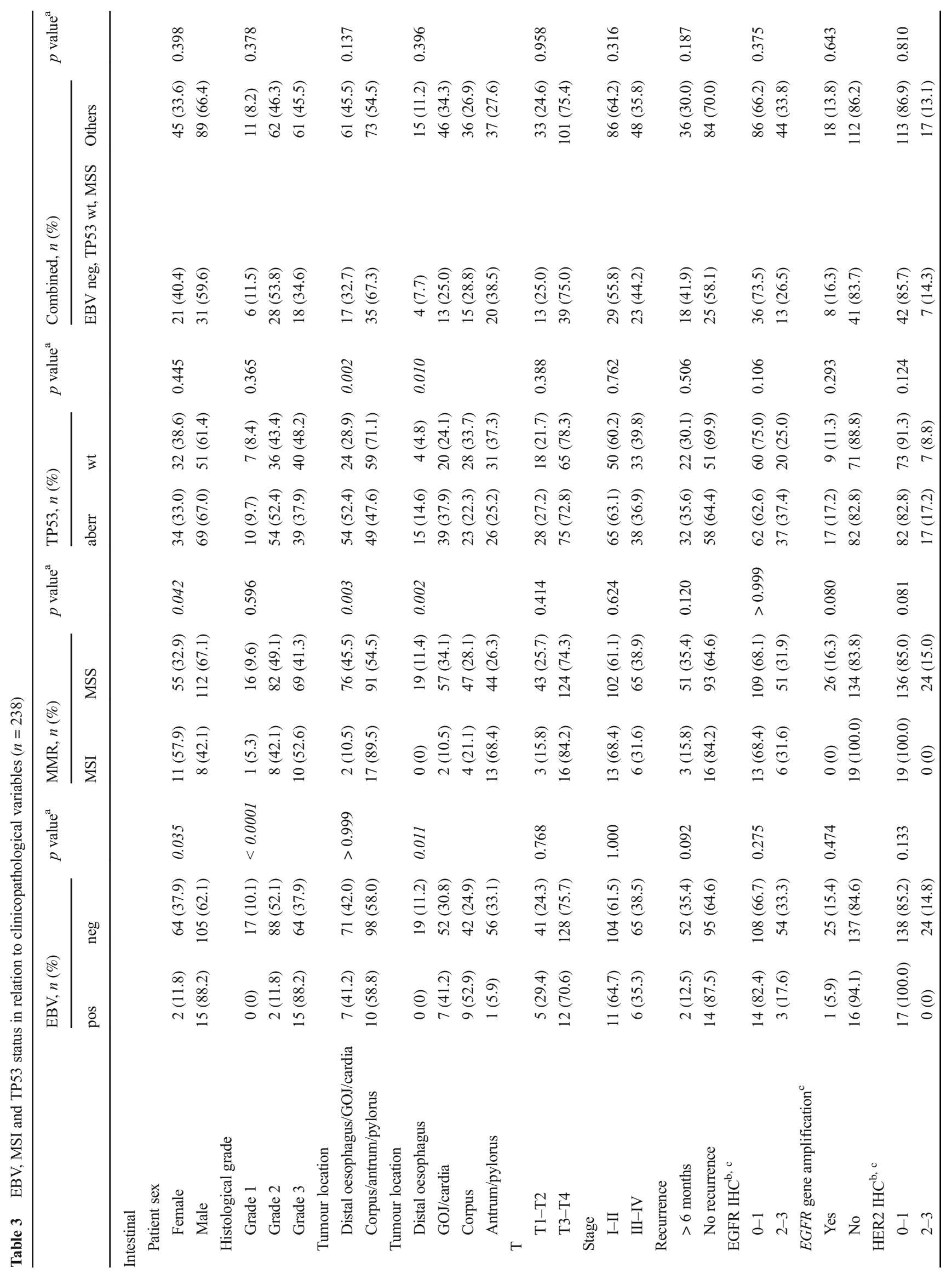




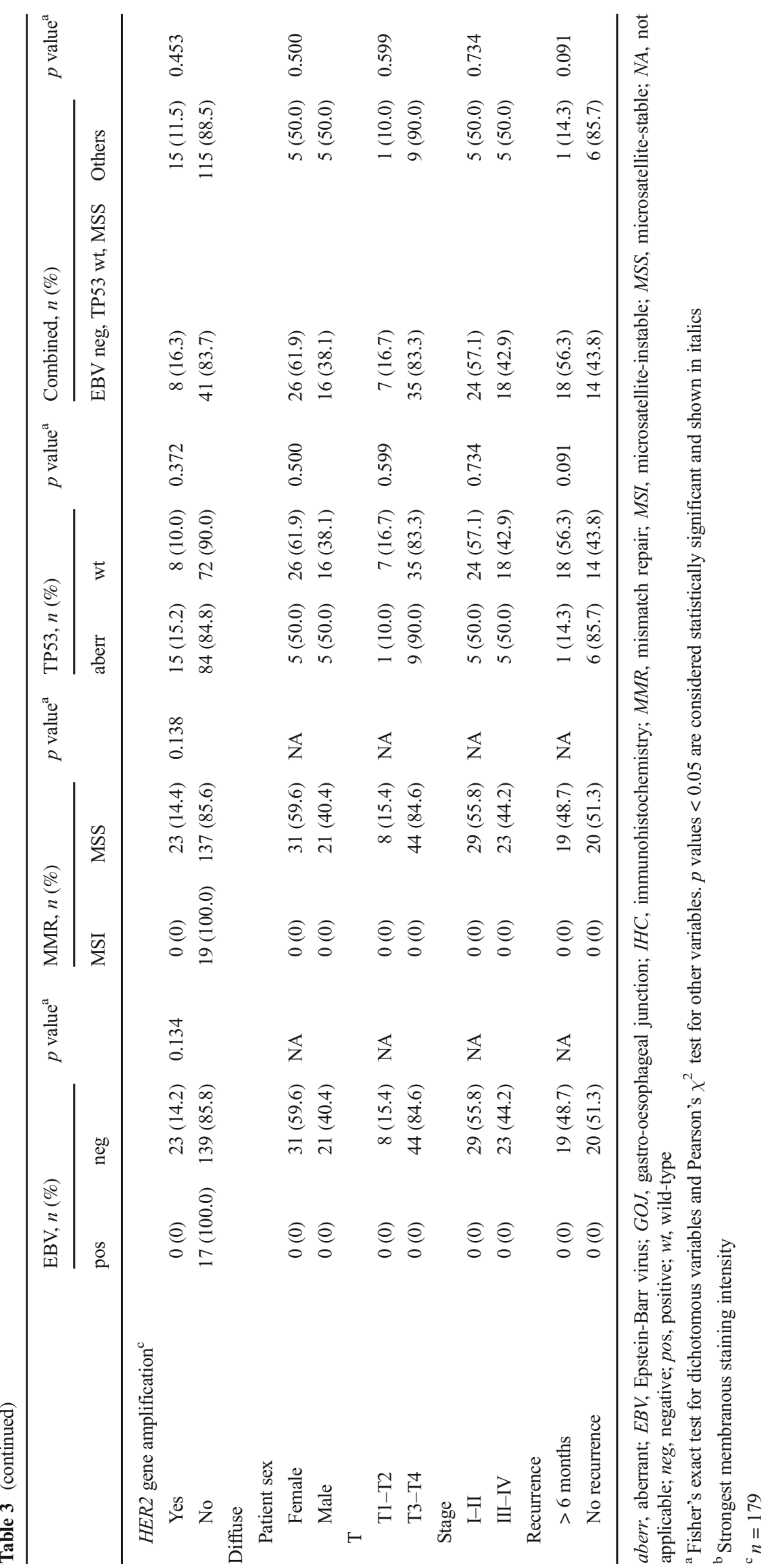




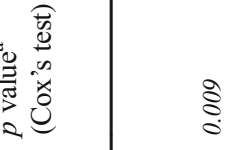

$\stackrel{\substack{+0}}{\stackrel{0}{0}}$

$\stackrel{2}{\vdots}$

$\infty$
$\infty$
+
$\stackrel{+}{+}$
$\stackrel{7}{+}$

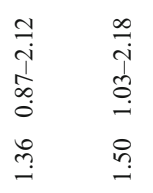

$\infty$
$\infty$
$\substack{\infty \\ 0}$

ind

言离离

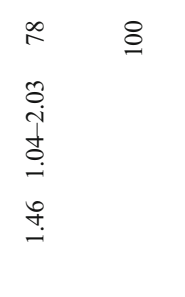

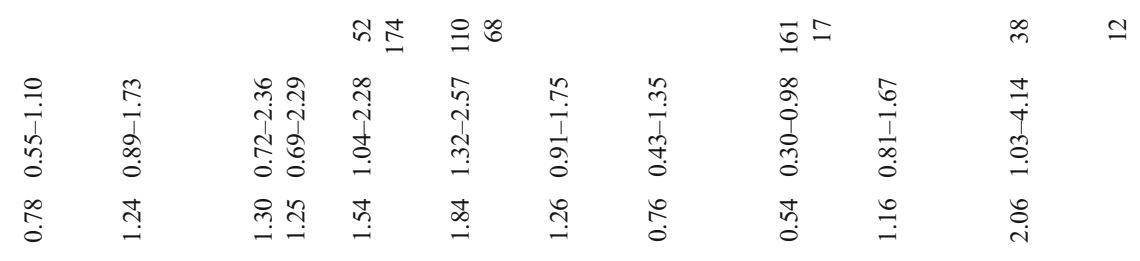

ลิ

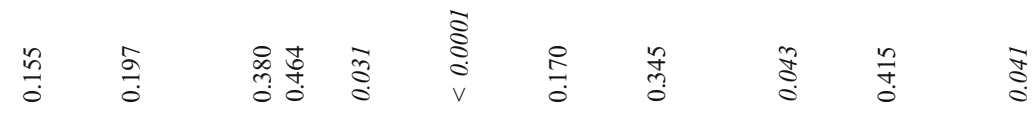

$\stackrel{0}{0}$

$\stackrel{n}{\circ} \frac{\circ}{0}$

․

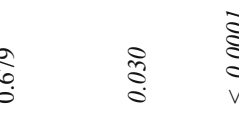

$\stackrel{8}{\circ}$

寺然

$\stackrel{8}{8}$

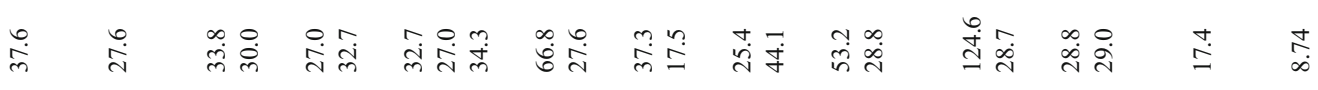

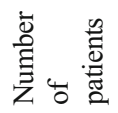
$\therefore \quad \cong$

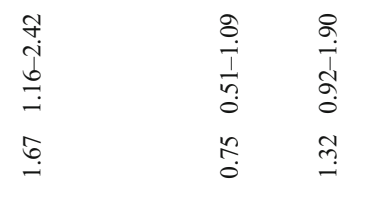
I文年

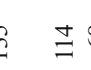
a

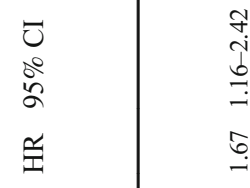
$\hbar \cong n g=\infty$

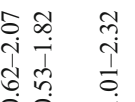

$\stackrel{m}{\sharp}$

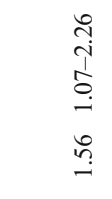

$\stackrel{\infty}{\infty}$
$\stackrel{+}{+}$
$\vdots$
0
0

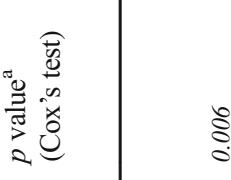

$\stackrel{8}{8}$

$\stackrel{m}{0} \quad \frac{7}{0}$

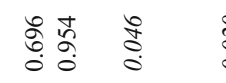

$\stackrel{\circ}{\circ} \frac{\circ}{0}$

$\stackrel{0}{\circ}$

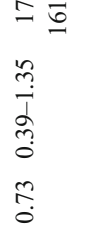

$=\underset{\sigma}{9} \stackrel{\infty}{\simeq}$

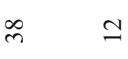

ڤ 를

2.

$\stackrel{8}{8}$

$\stackrel{0}{0} \stackrel{\infty}{0}$

ì

ลे $\frac{\infty}{0}$

ஓे

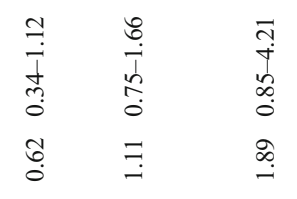

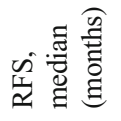

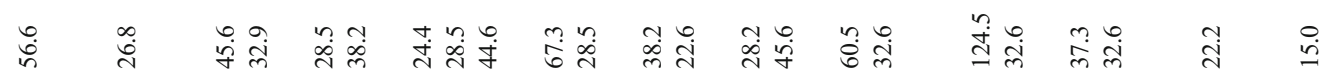
离

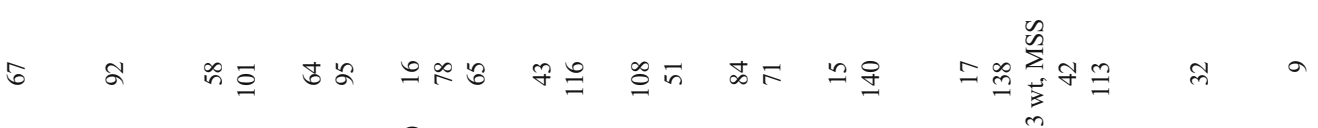

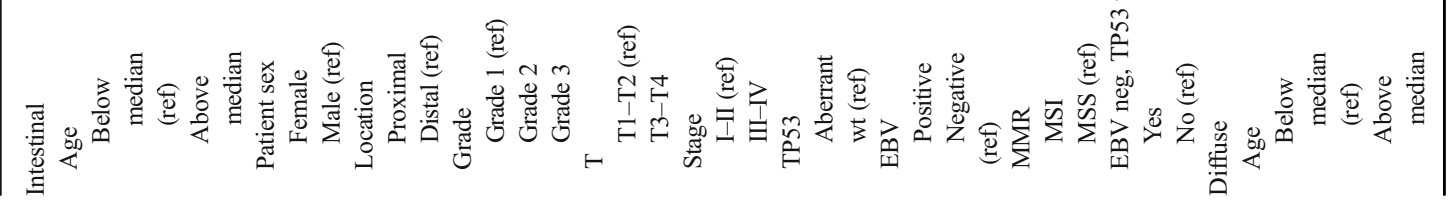




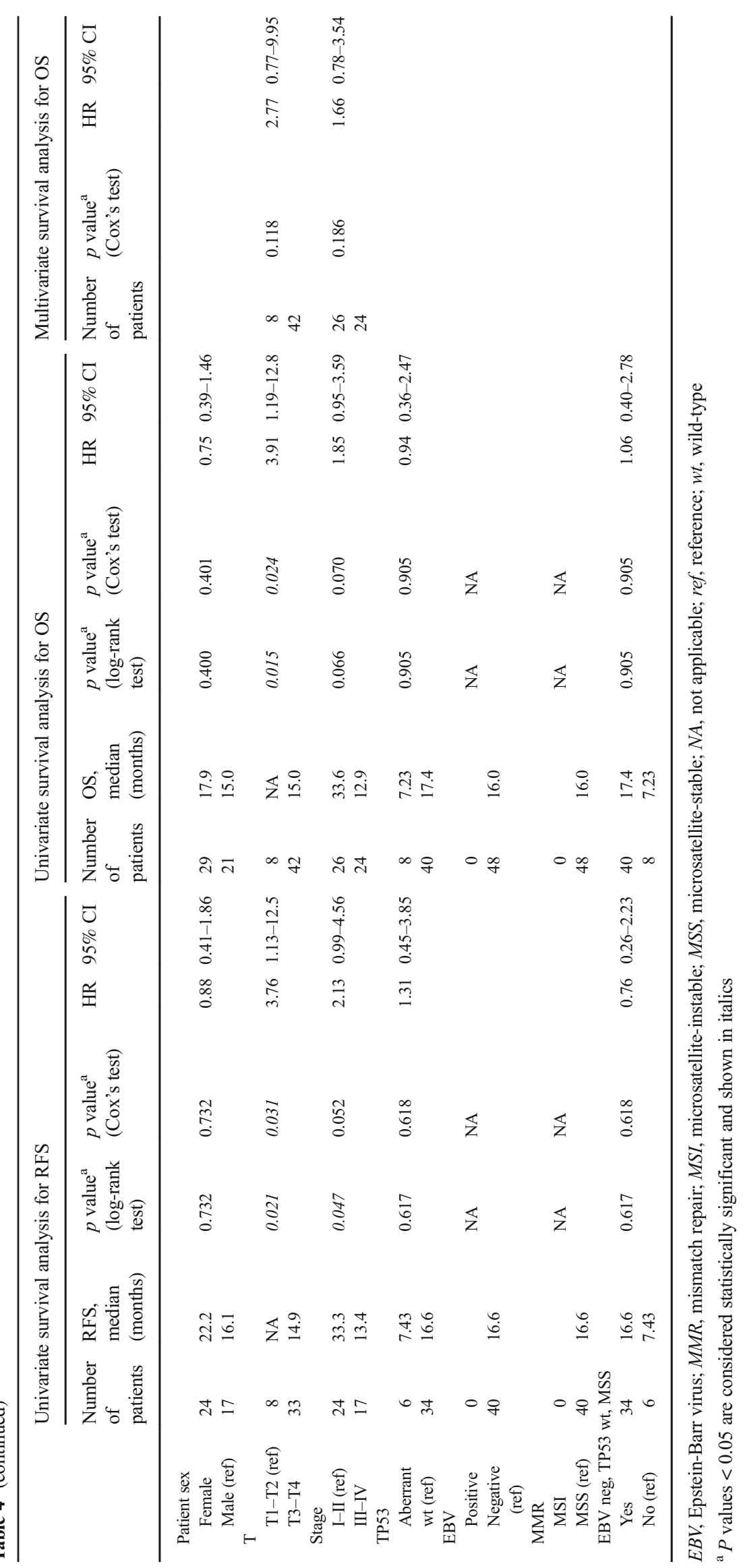


histological subtype can be used as a starting point for a clinically applicable method for molecular classification.

Among the diffuse-type tumours, the comparison of our results to the other studies should be considered as suggestive as we have analysed only a subset of the diffuse-type tumours diagnosed during the study period. The frequency of EBV positivity has been reported to be $3.8-9.8 \%$ among diffusetype tumours $[3,4,6,8]$, but in our study none of the diffusetype tumours was found to contain EBV positivity. Similarly, we did not detect MSI in the diffuse-type tumours. In other studies, the proportion of MSI diffuse-type tumours has been reported to be relatively low $(3.8-8.7 \%)[3,6]$ with the exception of $16.9 \%$ by Cristescu et al. (2015). The frequency of aberrant TP53 expression in our study was $19.2 \%$ among the diffuse-type tumours, which is quite similar to the observed frequencies of $26.1-27.5 \%$ [3, 4] but notably different from $53.8 \%$ reported by Kim et al. (2016).

The subgroup of EBV negative, MSS and wild-type TP53 tumours comprised $28.0 \%$ (52/186) of the intestinal-type tumours and $80.8 \%$ (42/52) of the diffuse-type tumours in our study population. In the TCGA study, this pattern was found in 23.0\% (45/196) of the intestinal-type and 56.5\% (39/69) of the diffuse-type tumours. In the ACRG study, the respective frequencies are $38.0 \%$ (57/150) for the intestinal-type tumours and $45.8 \%$ (65/142) for the diffuse-type tumours.

We could demonstrate the association of EBV positivity with male patients and the location of the tumour in the gastric corpus as previously shown by Ahn et al. (2017). Additionally, EBV positivity was found to be associated with poor histological differentiation among the intestinal-type tumours. Distally located tumours were more often characterised by MSI than proximal tumours, which confirms the results obtained by the TCGA and Cristescu et al. (2015). We could also show that $E G F R$ and HER2 gene amplifications were most common among the intestinal-type tumours with EBV negativity, MSS and TP53 aberration, of which 17.3\% (17/98) were EGFR and $15.3 \%$ (15/98) HER2 amplified. Notably, also among the EBV negative, MSS and TP53 wild-type tumours, the proportion of these amplifications, $15.4 \%(8 / 52)$ for both genes, was substantial. In the TCGA study population, $10.6 \%(9 / 85)$ of the EBV negative, MSS and TP53 aberrant tumours contained EGFR and 34.1\% (29/ $85)$ contained HER 2 gene amplification. Similar to our results, among the EBV negative, MSS and TP53 wild-type tumours, the proportion of $E G F R$ gene amplification was $11.1 \%(5 / 45)$ and HER 2 gene amplification $13.3 \%(6 / 45)[3,16,17]$. In our study, the co-localisation of aberrant TP53 expression together with $E G F R$ or HER2 gene amplification was noticed to be more common in the proximally than distally located intestinal-type tumours, which is in line with the results from a recent characterisation of oesophageal carcinomas showing strong genomic similarities between oesophageal adenocarcinomas and CIN-type gastric adenocarcinomas according to the TCGA classification [18]. Additionally, we observed that patients with MSI tumours had longer survival than patients with MSS tumours both in the univariate and multivariate analysis, which is consistent with earlier findings $[4,7]$.

Our classification method concentrated on the intestinal subtype of gastric adenocarcinoma, where receptor tyrosine kinase (RTK) copy number alterations are more common than in diffuse-type tumours. In the TCGA study population, all tumours with either EGFR or HER2 gene amplification were observed to have intestinal-type histology [3]. However, we also examined a subset of diffuse-type tumours in order to make comparisons with the results obtained by other studies. Most likely, due to this selection, we did not find EBV positivity or MSI among the diffuse-type tumours in our study population.

In order to increase the reliability of the TMA analysis, we have examined four tissue cores from each tumour. For the EGFR and HER2 SISH, we have used whole slide sections in order to account for the potential spatial heterogeneity of the gene amplifications. Additionally, we have examined the expression of all four MSI markers instead of only one in order to increase the reliability of the classification into MSI or MSS tumours. Especially in the MSI classification, there is variation in the reported prevalence of MSI phenotype, which may partly be related to technical issues in performing and interpreting the IHC reactions. Moreover, some variability is inevitably related to using TP53 IHC as a surrogate marker for TP53 gene mutations.

Our classification method differs from that suggested by Park et al. (2016) in that our initial division was based on the Laurén classification. After that, we continued by categorising the tumours first by EBV and second by MSI reactivity. Finally, we considered the presence of TP53 aberrations.

Our method was able to determine four distinct subgroups among the intestinal-type tumours without considerable overlapping of the molecular markers. Among the diffuse-type tumours in our material, EBV positivity, MSI and aberrant TP53 expression were mutually exclusive. Among the intestinal tumours, only one EBV positive tumour showed aberrant TP53 expression and four tumours with MSI showed aberrant TP53 expression. EBV positivity and MSI were mutually exclusive also among the intestinal-type tumours, which is in line with other studies [3,6]. Only 3/183 intestinal-type tumours $(1.6 \%)$ had aberrant E-cadherin expression, and even they could be classified as either EBV positive, MSI or TP53 aberrant. The proportion is comparable to the observed $4.1 \%$ (8/196) of the intestinal-type tumours with E-cadherin mutations in the TCGA study.

A few articles have recently been published describing different algorithms implementing the molecular classification system proposed by TCGA into clinical practice [5-8]. However, only few studies have utilised the Laurén 
classification and none of them have based their proposed algorithm on the histological subtype of the tumours. In one study, differentially expressed genes were compared by microarray analysis between intestinal and diffuse gastric cancers, and a 40-gene signature was created to serve as a prognostic tool [19]. As RTK amplifications are known to be more prevalent among the intestinal-type tumours, the histological subtype could be a relevant factor to take in to account when investigating new RTK-targeting therapies for gastric cancer [3]. In only one of these recent studies, the evaluation of both $E G F R$ and HER2 gene amplification status has been performed by ISH [7]. However, the SISH procedure was carried out on TMA slides, which potentially does not account for the tumour heterogeneity. So far, anti-EGFR antibodies have not shown survival benefit in phase III trials including gastric or oesophagogastric cancer patients $[20,21]$. One reason for this may be that the screening methods used have not been able to identify the subgroup of patients possibly responsive to anti-EGFR treatment. In future clinical trials, the application of new classification methods combining both histological and molecular information may become necessary in order to improve the clinical benefit obtained by new targeted therapies.

In conclusion, we have demonstrated that gastric adenocarcinomas can be classified into biologically and clinically relevant subgroups by using a straightforward and clinically applicable method based on the Laurén classification together with immunohistochemistry and in situ hybridisation.

Acknowledgements This study was supported by Finnish cancer organisations and Turku University Foundation. We thank Mr. Jaakko Liippo for help with the pictures.

Compliance with ethical standards The study was conducted in accordance with the Declaration of Helsinki and the Finnish legislation for the use of archived tissue specimens and associated clinical information. The clinical data were retrieved, and the histological samples were collected and analysed with the endorsement of the National Authority for Medico-Legal Affairs and The Ethics Committee of the Hospital District of Southwest Finland as well as with the permission of Auria Biobank hosting the specimen archive. All the specimens were from Auria Biobank, which has obtained its archived diagnostic sample collection with an opt-out procedure according to the Finnish biobank act [22]. Biobanks authorised and inspected by National Supervisory Authority for Welfare and Health can provide human specimens collected during diagnostic procedures and associated clinical information for research purposes based on the biobank's scientific board review. Thus, informed consent from surviving patients was not required.

Conflict of interest A $\mathrm{A}, \mathrm{ML}, \mathrm{RR}, \mathrm{JS}$ and $\mathrm{OC}$ are inventors in a patent related to this work: US 20110217296 A1 Method for selecting patients for treatment with an EGFR inhibitor. All remaining authors declare that they have no conflict of interest.
Open Access This article is distributed under the terms of the Creative Commons Attribution 4.0 International License (http:// creativecommons.org/licenses/by/4.0/), which permits unrestricted use, distribution, and reproduction in any medium, provided you give appropriate credit to the original author(s) and the source, provide a link to the Creative Commons license, and indicate if changes were made.

\section{References}

1. Ferlay J, Soerjomataram I, Ervik M, Dikshit R, Eser S, Mathers C, Rebelo M, Parkin D, Forman D, Bray F (2013) GLOBOCAN 2012 v1.0, cancer incidence and mortality worldwide: IARC CancerBase No. 11. Lyon, International Agency for Research on Cancer http:// globocan.iarc.fr. Accessed on 23 April 2017

2. Laurén P (1965) The two histological main types of gastric carcinoma: diffuse and so-called intestinal-type carcinoma. An attempt at a histoclinical classification. Acta Pathol Microbiol Scand 64:31-49

3. Cancer Genome Atlas Research Network (2014) Comprehensive molecular characterization of gastric adenocarcinoma. Nature 513: 202-209. https://doi.org/10.1038/nature13480

4. Cristescu R, Nebozhyn M, Kim K, Ting J, Wong S, Liu J, Yue Y, Wang J, Yu K, Ye X, Do I, Liu S, Gong L, Fu J, Jin J, Choi M, Sohn T, Lee J, Bae J, Kim S, Park S, Sohn I, Jung S, Tan P, Chen R, Hardwick J, Kang W, Ayers M, Hongyue D, Reinhard C, Loboda A, Aggarwal A (2015) Molecular analysis of gastric cancer identifies subtypes associated with distinct clinical outcomes. Nat Med 21:449-456. https://doi.org/10.1038/nm.3850

5. Setia N, Agoston A, Han H, Mullen J, Duda D, Clark J, Deshpande V, Mino Kenudson M, Srivastava A, Lennerz J, Hong T, Kwak E, Lauwers G (2016) A protein and mRNA expression-based classification of gastric cancer. Mod Pathol 29:772-784. https://doi.org/10. 1038/modpathol.2016.55

6. Kim HS, Shin S, Beom S, Jung M, Choi Y, Son T, Kim H, Cheong J, Hyung W, Noh S, Park J, Shin S, Lee S, Lee Y, Koom W, Lim J, Chung H, Rha S (2016) Comprehensive expression profiles of gastric cancer molecular subtypes by immunohistochemistry: implications for individualized therapy. Oncotarget 7:44608-44620. 10. 18632/oncotarget.10115

7. Park C, Park J, Kim H, Rha S, Hyung W, Cheong J, Noh S, Lee S, Lee Y, Huh Y (2016) Receptor tyrosine kinase amplified gastric cancer: Clinicopathologic characteristics and proposed screening algorithm. Oncotarget 7:72099-72112. 10.18632/oncotarget.12291

8. Ahn S, Lee S, Kim Y, Kim A, Shin N, Choi K, Lee C, Huh G, Kim K, Setia N, Lauwers G, Park D (2017) High-throughput protein and mRNA expression-based classification of gastric cancers can identify clinically distinct subtypes, concordant with recent molecular classifications. Am J Surg Pathol 41:106-115. https://doi.org/10. 1097/PAS.0000000000000756

9. Zlobec I, Suter G, Perren A, Lugli A (2014) A next-generation tissue microarray (ngTMA) protocol for biomarker studies. J Vis Exp (91):51893. https://doi.org/10.3791/51893

10. Bosman F, Carneiro F, Hruban R, Theise N (2010) WHO classification of tumours of the digestive system, 4th edn. IARC Press, Lyon

11. Altman DG, McShane LM, Sauerbrei W, Taube SE (2012) Reporting recommendations for tumor marker prognostic studies (REMARK): explanation and elaboration. BMC Med 10:51. https://doi.org/10.1186/1741-7015-10-51

12. Ålgars A, Lintunen M, Carpén O, Ristamäki R, Sundström J (2011) EGFR gene copy number assessment from areas with highest EGFR expression predicts response to anti-EGFR therapy in 
colorectal cancer. Br J Cancer 105:255-262. https://doi.org/10. 1038/bjc.2011.223

13. Rüschoff J, Hanna W, Bilous M, Hofmann M, Osamura RY, Penault-Llorca F, van de Vijver M, Viale G (2012) HER2 testing in gastric cancer: a practical approach. Mod Pathol 25:637-650. https://doi.org/10.1038/modpathol.2011.198

14. Ålgars A, Avoranta T, Österlund $\mathrm{P}$, Lintunen M, Sundström J, Jokilehto T, Ristimäki A, Ristamäki R, Carpén O (2014) Heterogeneous EGFR gene copy number increase is common in colorectal cancer and defines response to anti-EGFR therapy. PLoS One 18, 9(6):e99590. https://doi.org/10.1371/journal.pone.0099590

15. Birkman E-M, Ålgars A, Lintunen M, Ristamäki R, Sundström J, Carpén O (2016) EGFR gene amplification is relatively common and associates with outcome in intestinal adenocarcinoma of the stomach, gastro-oesophageal junction and distal oesophagus. BMC Cancer 16:406. https://doi.org/10.1186/s12885-016-2456-1

16. Cerami E, Gao J, Dogrusoz U, Gross B, Sumer S, Aksoy B, Jacobsen A, Byrne C, Heuer M, Larsson E, Antipin Y, Reva B, Goldberg A, Sander C, Schultz N (2012) The cBio cancer genomics portal: an open platform for exploring multidimensional cancer genomics data. Cancer Discov 2:401-404. https://doi.org/10. 1158/2159-8290.CD-12-0095

17. Gao J, Aksoy B, Dogrusoz U, Dresdner G, Gross B, Sumer SO, Sun Y, Jacobsen A, Sinha R, Larsson E, Cerami E, Sander C, Schultz N (2013) Integrative analysis of complex cancer genomics and clinical profiles using the cBioPortal. Sci Signal 6:pl1. https://oi. org 1 10.1126/scisignal.2004088

18. Cancer Genome Atlas Research Network (2017) Integrated genomic characterization of oesophageal carcinoma. Nature 541:169175. https://doi.org/10.1038/nature20805

19. Min L, Zhao Y, Zhu S, Qiu X, Cheng R, Xing J, Shao L, Guo S, Zhang $S$ (2017) Integrated analysis identifies molecular signatures and specific prognostic factors for different gastric cancer subtypes. Transl Oncol 10:99-107. https://doi.org/10.1016/j.tranon.2016.11.003

20. Lordick F, Kang Y, Chung H, Salman P, SC O, Bodoky G, Kurteva G, Volovat C, Moiseyenko VM, Gorbunova V, Park JO, Sawaki A, Celik I, Götte H, Melezínková H, Moehler M (2013) Capecitabine and cisplatin with or without cetuximab for patients with previously untreated advanced gastric cancer (EXPAND): a randomised, openlabel phase 3 trial. Lancet Oncol 14:490-499. https://doi.org/10. 1016/S1470-2045(13)70102-5

21. Waddell T, Chau I, Cunningham D, Gonzalez D, Frances A, Okines C, Wotherspoon A, Saffery C, Middleton G, Wadsley J, Ferry D, Mansoor W, Crosby T, Coxon F, Smith D, Waters J, Iveson T, Falk S, Slater S, Peckitt C, Barbachano Y (2013) Epirubicin, oxaliplatin, and capecitabine with or without panitumumab for patients with previously untreated advanced oesophagogastric cancer (REAL3): a randomised, open-label phase 3 trial. Lancet Oncol 14:481-489. https://doi.org/10.1016/S1470-2045(13)70096-2

22. Finlex Data Bank. https://www.finlex.fi/fi/laki/kaannokset/2012/ en20120688.pdf. Accessed 23 April 2017 